\title{
Reflets
}

Revue ontaroise d'intervention sociale et communautaire

\section{Se définir et se renouveler en dépit des contraintes}

\section{Madeleine Dubois et Marie-Luce Garceau}

Volume 6, numéro 1, printemps 2000

Approches d’intervention : définir et renouveler nos pratiques

URI : https://id.erudit.org/iderudit/026292ar

DOI : https://doi.org/10.7202/026292ar

Aller au sommaire du numéro

Éditeur(s)

Reflets : Revue ontaroise d'intervention sociale et communautaire

ISSN

1203-4576 (imprimé)

1712-8498 (numérique)

Découvrir la revue

Citer cet article

Dubois, M. \& Garceau, M.-L. (2000). Se définir et se renouveler en dépit des contraintes. Reflets, 6(1), 10-15. https://doi.org/10.7202/026292ar

Tous droits réservés (C) Reflets : Revue ontaroise d'intervention sociale et communautaire, 2000
Ce document est protégé par la loi sur le droit d'auteur. L'utilisation des services d'Érudit (y compris la reproduction) est assujettie à sa politique d'utilisation que vous pouvez consulter en ligne.

https://apropos.erudit.org/fr/usagers/politique-dutilisation/ 


\section{Se définir et se renouveler en dépit des contraintes}

\section{Madeleine Dubois et Marie-Luce Garceau}

En lançant l'appel de contributions pour le présent numéro sous le thème "Approches d'intervention : définir et renouveler nos pratiques», notre but était de présenter un numéro orienté plus spécifiquement vers un des objectifs de Reflets, soit celui d'approfondir la réflexion et l'analyse sur les pratiques. Comme ce numéro marque notre cinquième anniversaire de publication, il nous semblait important de créer un espace pour consigner et relater certaines de nos pratiques, afin de faire émerger des orientations, des perspectives et de donner un sens à des expériences qui nous semblent parfois uniques ou singulières.

Au cours des cinq dernières années, nous savons qu'occasionnellement, la lecture d'un article dans Reflets au sujet de ressources ou de projets existants dans un coin de la province a donné lieu à des échanges de renseignements avec des intervenantes et intervenants d'autres régions et ce, afin d'évaluer la création possible d'une ressource ou d'un projet semblable dans sa région. En s'inscrivant dans cette même veine qui dénote le besoin de partage et de réflexions sur nos pratiques, nous souhaitons que ce numéro suscite une fois de plus une prise de conscience de la signification et de la portée des pratiques sociales et communautaires en Ontario français, tant chez les intervenantes et les intervenants que chez les chercheures et les chercheurs.

Parler d'articulation et de renouvellement des pratiques dans une province aussi diversifiée que la nôtre en termes géographique, de besoins ou de ressources, mène à l'exploration de sujets variés et soulève un questionnement sur la signification même du 
concept de renouvellement, dans le contexte sociopolitique et économique actuel.

Miroirs des réalités multiples qui se présentent, les articles de ce numéro logent à plusieurs enseignes qui sont autant des pistes de réflexion orientées sur des concepts théoriques ou discursifs, sur le questionnement de pratiques et de services, sur le besoin de développer des ressources et, finalement, sur l'action sociale et les pratiques de résistance.

Ainsi, à partir d'une réflexion sur la théorie de l'étiquetage et de son application à plusieurs champs de pratique, l'article de St-Amand nous invite à questionner les appellations couramment utilisées pour nommer les personnes auprès de qui nous intervenons, notamment celles ayant un vécu psychiatrique. S'intéressant aussi à ces personnes, Lemieux montre comment un programme visant à offrir un meilleur soutien et un entourage élargi aux personnes psychiatrisées afin de les maintenir dans la communauté et éviter de recourir à une hospitalisation qui n'est pas toujours requise. Toujours dans le domaine de la santé mentale, Leclerc évoque la nécessité de raffiner nos outils afin d'éviter la dissimulation diagnostique qui consiste à camoufler les troubles psychologiques derrière le handicap intellectuel.

Sur le plan de ce qu'on pourrait nommer des pratiques de résistance ou d'action sociale, les articles de Boudreau et Nabigon et celui de Gouault présentent des luttes pour parvenir à l'autodétermination ou à l'autonomie. Alors que le premier dresse le portrait des luttes menées par les Premières Nations, le second relate, à travers l'histoire d'un centre d'alphabétisation, comment les personnes acquièrent une estime de soi et une confiance en elle, nécessaire à leur autonomie et leur participation pleine et entière à la société.

De par leur contenu et leurs propos, l'ensemble des articles nous entraîne dans une réflexion sur la signification et la portée $\mathrm{du}$ vocable de renouvellement, dans un contexte de rationalisations et de compressions, où le social se voit imposer une logique d'efficacité qui occulte et subvertit souvent les besoins et les intérêts, individuels et collectifs. Dans un tel contexte, l'idée même de renouveler nos pratiques doit se concevoir en termes fort 
modestes. Par exemple, elle peut correspondre au développement de mécanismes ou des stratégies pour composer avec les logiques centralisatrices de l'État qui ne répondent pas aux besoins des régions éloignées ou rurales, à la lutte pour assurer le maintien de certains acquis et certaines ressources pour nos communautés francophones ou encore, à la recherche de brèches dans des logiques d'organisation de services visant davantage à répondre aux besoins du système que ceux des individus et des communautés. Dans tous ces cas, il s'agit, dans la mesure du possible, de pouvoir garder le cap sur les besoins humains plutôt que sur des logiques plus efficaces de contrôle et d'administration.

À leur manière, les articles du présent numéro ouvrent des brèches sur des stratégies et des pratiques visant à améliorer les conditions sociales, politiques ou économiques des francophones de l'Ontario et des diverses cultures faisant partie de cette francophonie. Ces stratégies s'expriment par le biais de luttes et de mouvements dont traitent les articles de St-Amand, de Gérôme, de Gouault et de Boudreau et Nabigon; par le développement de partenariats - les luttes ne pouvant se faire de façon isolée dont il est question, notamment dans les articles de Bélanger et van de Sande, de Dupont et al., de Leclerc et de Lemieux; par l'élaboration de formations visant, entre autres, la prise de conscience ou encore la nécessité de développer du matériel pédagogique adapté à la francophonie comme le souligne les articles de Garceau, de Dupras, de Dutheuil, de Champion et de Barreira.

Par ailleurs, certains articles invitent à une réflexion plus approfondie sur diverses facettes pouvant mener au renouvellement de nos pratiques. En effet, St-Amand démontre l'importance des mots que nous utilisons pour nommer les personnes utilisant les services et il nous met en garde contre la marginalisation que ces appellations entraînent pour les personnes, tout en démontrant de quelles façons ces vocables sont réductionnistes, diffamatoires et parfois irrévocables. Après avoir dégagé une typologie de l'aide à partir des façons dont nous désignons les personnes, il offre des pistes pour réfléchir, voire renouveler notre vocabulaire. 
S'inscrivant aussi dans une perspective de mise en question, à la fois de nos biais personnels et professionnels, l'article de Boudreau et Nabigon montre comment certaines pratiques et politiques assimilationnistes à l'égard des Premières Nations ont mené à la perte de leurs pouvoirs économique, social, politique et à la perte de leur culture. Ils montrent que l'autonomie gouvernementale des Premières Nations passe par la prise en main politique et le renouveau culturel informé de la spiritualité et offrent certaines clés pour repenser nos pratiques en se centrant sur les principes autochtones de la roue de guérison.

L'histoire mouvementée du programme d'alphabétisation offert à La Magie des lettres et relatée dans l'article de Gouault, illustre les multiples renouvellements auxquels a dû se prêter l'organisme au cours de ses 15 années d'existence pour s'adapter aux exigences de politiques d'éducation étroites et réductionnistes, tout en restant fidèle à ses orientations et à sa mission.

Renouveler, c'est aussi faire un bilan de nos pratiques pour voir les changements, les orientations à prendre et les mécanismes à mettre en place. Ces bilans donnent parfois lieu au développement de matériel plus approprié aux réalités de la francophonie; c'est ce que démontre l'article de Garceau qui présente une série de fiches créées dans le but de mieux faire connaitre les problématiques liées à la violence et de former de plus en plus de femmes afin qu'elles puissent intervenir. Le développement de matériel est aussi lié au besoin de formation, sujet sur lequel se penche Dupras dans un article sur les besoins de formation en matière de suicide.

L'article de Leclerc traite d'une problématique maintes fois relevée lorsqu'il s'agit de faire des bilans des services et des ressources s'adressant aux francophones, soit celle de manques ou de lacunes dans les services pour certains groupes de personnes. Il souligne aussi que le renouvellement des pratiques doit forcément s'appuyer sur des recherches rigoureuses, sur la formation interdisciplinaire et favoriser la collaboration entre les services afin de mieux répondre aux besoins des populations ciblées.

Ce thème de développement de services répondant aux besoins particuliers d'une population est repris par Lemieux qui traite du 
développement de services d'accompagnement dans une région sous desservie à cet effet. Après avoir montré ce que signifie une situation de crise pour les personnes vivant avec un handicap psychiatrique, elle définit les sphères des besoins fondamentaux auxquels les bénévoles peuvent répondre et présente un modèle de formation pour les personnes qui accompagneront les personnes en crise.

Face aux coupures et aux restrictions budgétaires drastiques, le partenariat devient souvent une condition sine qua non au renouvellement des pratiques. Dupont et al. montrent comment les groupes de femmes travaillant dans le domaine de la violence et de l'agression contre les femmes se voient obligées de trouver des partenaires afin de pouvoir continuer à développer l'ensemble des services nécessaires.Vision sobre du partenariat qui démontre que malgré ses aspects positifs, il peut s'agir d'une expérience exigeante et que les femmes doivent se donner des critères de base à respecter dans le développement de ceux-ci.

Champion et Barreira nous renvoient aussi à l'utilité et au bien-fondé du partenariat dans un article sur la collaboration entre une école élémentaire, son personnel enseignant, les parents et leurs enfants et l'École de sciences infirmières, dans un projet visant à encourager les jeunes et leurs parents à faire des activités physiques ensemble. Quant à Plante, elle présente les jalons ayant mené à l'établissement d'un partenariat entre les institutions publiques, les écoles et les organismes communautaires pour répondre à des besoins de formation en matière de sexualité pour les enfants et leurs parents. Tout en soulignant l'importance de partenariats dans la lutte contre la pauvreté des enfants, Bélanger et van de Sande introduisent un autre défi dont on doit tenir compte en réfléchissant au renouvellement des pratiques: celui de développer une analyse et des pistes d'action adaptées aux particularités régionales. Ces auteurs montrent l'accroissement de la pauvreté chez les enfants et les jeunes dans un Sudbury qui nous semblait pourtant pouvoir répondre aux besoins de sa population. En fait, s'il est une région dans laquelle le taux de pauvreté dépasse celui des échelles nationales ou provinciales, c'est bien celle du Grand Sudbury. Les recommandations d'action 
proposées peuvent n'avoir de signification que dans la mesure où elles sont ancrées dans les spécificités de la région et où elles suscitent l'engagement de tous les partenaires communautaires.

En s'appuyant sur des cas précis pour montrer certaines difficultés inhérentes à la communication entre personnes de différentes cultures, Sterlin et Dutheuil mettent en lumière l'importance de la décentration et de l'ouverture à d'autres cultures dans la quête de renouvellement. Ils offrent des pistes pour éviter, voire éliminer le contre-transfert culturel entre autres, en discutant de l'adoption d'une vision cosmocentrique, c'est-à-dire une vision d'un monde où chacun à une place malgré les différences, pour que nous puissions mieux intervenir auprès des personnes ou des groupes ethniques et pour que nous puissions utiliser la communication comme un apprentissage vers la coexistence.

Et finalement, le renouvellement constitue souvent une invitation à faire des retours dans l'histoire pour mieux comprendre vers où l'on veut se diriger. Dans l'entrevue avec Roland Lecomte, ancien directeur de l'École de service social de l'Université d'Ottawa et de celle de l'Université Carleton, nous vous offrons une perspective historique de l'évolution du service social au cours des quarante dernières années.

Que ce soit les années d'expérience et la sagesse accumulée de Roland Lecomte, les trente ans de pratique de Nérée St-Amand, les quinze ans d'existence de La Magie des lettres, l'insistance de chercheurs alliés à des groupes pour éveiller, depuis plus de dix ans, les politiciens locaux au problème de la pauvreté des enfants à Sudbury, tout concorde pour indiquer une évidence, presque qu'une lapalissade, qu'on omet trop souvent de dire : une pratique pour se renouveler doit durer, s'inscrire dans le temps. Il ne s'agit cependant pas d'un temps de reproduction au Même, de consécration à l'Identique ou de «routinisation» de l'existence, mais d'un temps engageant à la réflexivité, pour reprendre l'expression de Giddens ou de Touraine. L'historicité ou l'inscription dans l'histoire s'accompagne d'un retour sur soi, sur sa pratique pour conforter son identité, y constater ses échecs et ses réussites, afin ultérieurement de s'ouvrir au monde et de voir le monde autrement. Le renouvellement des pratiques est au prix d'un enracinement nous permettant d'aller ailleurs. 